\title{
On the Impact of Robotics in Behavioral and Cognitive Sciences: From Insect Navigation to Human Cognitive Development
}

\author{
Pierre-Yves Oudeyer
}

\begin{abstract}
The interaction of robotics with behavioral and cognitive sciences has always been tight. As often described in the literature, the living has inspired the construction of many robots. Yet, in this article, we focus on the reverse phenomenon: building robots can impact importantly the way we conceptualize behavior and cognition in animals and humans. This article presents a series of paradigmatic examples spanning from the modelling of insect navigation, the experimentation of the role of morphology to control locomotion, the development of foundational representations of the body and of the self/other distinction, the self-organization of language in robot societies, and the use of robots as therapeutic tools for children with developmental disorders. Through these examples, I review the way robots can be used as operational models confronting specific theories to reality, or can be used as proof of concepts, or as conceptual exploration tools generating new hypotheses, or used as experimental set ups to uncover particular behavioral properties in animals or humans, or even used as therapeutic tools. Finally, I discuss the fact that in spite of its role in the formation of many fundamental theories in behavioral and cognitive sciences, the use of robots is far from being accepted as a standard tool and contributions are often forgotten, leading to regular rediscoveries and slowing down cumulative progress. The article concludes by highlighting the high priority of further historical and epistemological work.
\end{abstract}

Index Terms-Behavioral and cognitive sciences, development, embodiment, epistemology, modelling, robotics, self-organization, therapeutic tools.

\section{INTRODUCTION}

$\mathbf{T}$ HE OBJECTIVE of this article is to show that experiments and models based on the use of robots can change the way we understand behavior and cognition in various profound ways. Many survey articles describe thoroughly how biology and psychology can inspire the building and programming of robots [4], [6], [10], [15], [36], [52]. On the contrary, while there has been many technical specific articles in the past presenting experiments that changed our understanding of given behavioral or cognitive phenomenon, few synthetic, concise, and interdisciplinary overviews exist that specifically focus on the potential impact of robotics in behavioral and cognitive sciences, and its conceptualization.

Manuscript received January 14, 2009; revised November 15, 2009. Date of publication January 08, 2010; date of current version March 12, 2010.

The author is with INRIA Bordeaux Sud-Ouest, France (e-mail: pierre-yves. oudeyer@inria.fr).

Color versions of one or more of the figures in this paper are available online at http://ieeexplore.ieee.org.

Digital Object Identifier 10.1109/TAMD.2009.2039057
Cordeschi [33] presents a thorough, technical, and comprehensive review targeted at specialists and historians. Clark [29], Boden [13], and Dennett [42], emphasize, among other topics, philosophical issues of the use of robots as tools for cognitive sciences. Other works such as [10], [36], [117], and [118] describe the tight interrelationships between robotics and behavioral and cognitive sciences, but do not specifically try to sort out and identify the specific structural properties of the impact of robotics in behavioral and cognitive sciences. Moreover, they remain rather technical and are targeted to a readership already knowledgeable in robotics.

Because a real long-term impact in behavioral and cognitive sciences can only be reached if behavioral and cognitive scientists are aware of and understand the work of roboticists, and because there are historical examples of loss of scientific efficiency due to this lack of awareness, synthetic, concise, and interdisciplinary studies focusing exclusively on what robotics may bring to behavioral and cognitive sciences should be helpful to establish firm bridges among these communities. Webb presented such an attempt [161], showing how robot models can be used to advance our understanding of insect-like behavior. This article tries to present a structured overview of how robots can help us better understand a wider spectrum of behavioral and cognitive phenomena, including for example, human locomotion, the development of the self/other distinction, language formation, or developmental cognitive and social disorders.

After the description of some historical roots, the article is structured around four broad types of potential contribution of robotics to behavioral and cognitive sciences. For each of these types, paradigmatic examples of robotic models or experiments are presented and discussed. The main criterion for choosing these examples was that they should be simple, widely understandable, and that the specific impact of robotics in behavioral cognitive sciences could be very clearly sorted out from the often concomitant engineering goal of trying to build robust or adaptive bio-inspired robots. As a consequence, while some of these examples are very recent and state-of-the-art, a number of other robotic models and experiments in this article have now been superseded by more advanced, but conceptually similar, models.

Furthermore, the examples chosen in this article focus on the specific impact, in behavioral and cognitive sciences, of having a robotic model as opposed to only a computational model: thus, in all of them the role of embodiment is central. As a consequence, all computational modeling work in which the use of robots is not central (even if present and necessary), and in par- 
ticular a very large computational neuroscience literature that has had a significant impact in brain sciences, has been left out. The interested reader may consult existing reviews in this field [3], [4].

\section{The Precursors: Robots, Biology, AND FolK PSYCHOLOGY IN THE FIRST HALF OF THE 20TH CENTURY}

In 1912, two engineers named John Hammond, Jr., and Benjamin Miessner built a robot which generated quite a stir in American media and in the scientific world [33]. This robot, called "Electric dog," was an electric wheeled machine which mechanisms had it orient towards light sources and track them with reactive and complex trajectories. Following the example of the "Electrical Experimenter" magazine, the press and the general public soon described this machine as "thinking," "nearly superhuman intelligence," and as "one of the most sensational inventions ever." The impact was even increased by the fact that Hammond and Miessner targeted a military application: they wanted to equip torpedos and missiles with this system so that they could automatically head on enemy antiaircraft batteries which used powerful light projectors during the night [105].

As a matter of fact, the underlying mechanism was relatively simple: Hammond and Miessner drew their inspiration from the work of famous biologist Jacques Loeb and his theories of phototropic behavior in certain kinds of insects, moths in particular. While the vitalist debate was still blustering, with defenders of the idea that an immaterial "vital principle" was needed to explain the behavior of living organisms [48], [98], and while other scientists like Alfred Binet, Francis Darwin, or Ernst Haeckel used anthropomorphic terminologies to describe the behavior of the simplest organisms by attributing them "will" or "consciousness" [33], Jacques Loeb proposed that at least certain behaviors could be explained by purely chemical and physical reflex mechanisms. In particular, he argued that the behavior of phototropic insects, which appear as complex trajectories around lights sources, could be explained simply by the fact that the muscles on the side of the animal that is struck by the light were more activated than those on the opposite side. Thus, Loeb proposed to consider these insects as pure "chemical heliotropic machines." This theory was presented in his book "Comparative Physiology of the Brain and Comparative Psychology" in 1900 [89]. Yet, its arguments were verbal and did not convince the scientific community, especially under the criticisms from the vitalists and from those who thought that a more complicated system was necessary to reproduce the behavior of these insects.

This explains why Loeb discovered with a great interest the machine that Hammond and Miessner built. They had managed to build an entire mechanical heliotropic machine, through a direct electromechanical coupling among light sensors and motors based on the principles stated by Loeb, which reproduced closely the behavior of phototropic insects. The "Electric dog" was proving the coherence and the plausibility of his theories, which so far were denied by the scientific community. In a subsequent book which had a much larger impact ("Forced Movements, Tropisms, and Animal Conduct”, 1918), Loeb presented in detail the robot and wrote: [it seems to me that] the effective building of an heliotropic machine does not only support mechanistic conceptions of voluntary and instinctive actions in animals, but also [my] theory on heliotropism, since this theory was used as a foundation to build this machine. [90:69].

Hammond and Miessner's robot was not only showing the plausibility and the coherence of Loeb's theory, it was also a severe blow towards vitalists and those who credited the simplest animals with "will," "consciouness," or "teleology." First of all, it was a clear proof that organized heliotropic behaviors could be reproduced solely with physical mechanisms: the vital principle was not necessary to explain certain behaviors of the living. Second, it showed that it was possible to generate a behavior that appeared voluntary and teleological, but which was not in practice. This was magnified by all the reactions and interpretations in the press and in the general public who spoke of a "nearly superhuman intelligence."

Besides, this last point identifies another contribution of the "Electric dog," which it shares with its successors, such as Ashby's homeostat [8] who was qualified as a "thinking machine," or Gray Walter's electric tortoise which caused a stir at the England festival in 1951 [158], or Arthur Samuel's checkers player which reached high TV audience on CBS in 1960 [130], or more recently the robotic football players of Robocup (http://www.robocup.org): the presence of these robots gave the opportunity to highlight certain cognitive biases of humans, in particular their tendency to anthropomorphise when they try to explain the phenomena they study, such as animal behavior. Thus, the "Electric dog" has been, even it is was initially unintentional, a tool that not only allowed researchers to study the plausibility of Loeb's theories and vitalism, but also permitted to observe the psychology of human observers, whether a scientists or not.

The story of the "Electric dog" was still an isolated case in the beginning of the 20th century, when the word "robot" did not even exist yet. Nevertheless, it is emblematic of the relations between robotics and biological, cognitive, and behavioral sciences that developed subsequently, and in particular in the last 20 years [33]. First, this relation shows the contribution that natural and behavioral sciences can provide to technology, robotics in particular. This is probably the most studied aspect of the relations between these domains [6], [52].

Yet, the other side of this interaction is as fundamental and is the topic of this article: Hammond and Miessner's work gives an insight on the way the construction of robots can profoundly impact the way scientists conceive the living, behavior, and intelligence. For the first time, important hypothesis can be tested experimentally, either by proving their internal coherence, or by proving their non-necessity or their sufficiency. Moreover, this construction forces researchers to formulate hypotheses more precisely and more completely. New hypotheses can also be the result of such a process of robot construction. In parallel, thanks to this experimental method and to the use of the artificial, the theorician's activity and his epistemology are called into question. The robot is not only a model, it is also an experimental setup for studying humans (or animals) that interact with him. We will now focus on more recent examples, and for each of 
them show how they illustrate one of these different types of contributions of robotics to behavioral and cognitive sciences.

\section{Robots AS OPERATIONAL MODELS CONFRONTING HYPOTHESES TO REALITY}

The impact of the "Electric dog" on biological sciences was a side effect of Hammond and Miessner's work, which objective was rather to take inspiration from Loeb's ideas to build more efficient machines. The two last decades of the 20th century witnessed the development of a whole set of robotics projects which directly aimed at evaluating hypotheses proposed by biologists [161]. Interestingly, many recent robotic models are related to the insects that passionated Loeb. Yet, 75 years later, the central question is no longer whether a vital principle was needed or not, but rather which particular physical and chemical mechanisms are responsible of these insects' behaviors [161].

Insect Navigation: For example, an important challenge is the understanding of the mechanisms that allow insects to fly in straight line, to fixate a visual objective, to track an objective, or to land [63], [136]. Indeed, insects are characterized by fixed focal eyes which cannot move, which prevent them to evaluate object distances through binocular vergence or through the refractive effort that is needed to see them sharply. A number of biologists proposed that they might use movement information, termed optical flow [51], [160]. Several indices computed from image movements, such as the global speed difference perceived by each eye, were proposed and first tested in experiments with flies and bees [135], [136]. In spite of encouraging results, they did not allow researchers to conclude that these optical flow indices alone could explain how bees navigate visually. Moreover, certain biologists had proposed that these indices could be computed using very simple neural reflex circuits, but it was rather speculative. Then, robots were constructed, in which the computation of movement indices was implemented and coupled with very simple control systems based on reflexes [131], [136]. It was shown that this allowed robots to navigate in the center of variable width and orientation corridors or tunnels in a way that beared close resemblance with bees [131]. Yet, it was also shown that the reflex mechanisms sometimes produced problematic behaviors in environments that contained untextured surface patches, and that it was necessary to add more complex mechanisms including short term memory to allow the navigation to be as robust as that of the bees. Thus, these robotic experiments showed that optical flow was informationally sufficient for bee-like visual navigation, but that pure reflex mechanisms were not sufficient to explain the robustness of navigation behaviors in bees and flies.

Even more specifically, other researchers tried to use robots to study the validity of physiological hypothesis about the neuronal circuits that implement navigation control in flies. Biologists proposed that the same simple neuronal structure was responsible for the stabilization of flight trajectories, stationary flight, and approach of stationary objects [59], and identified a set of neurons potentially involved in this circuit [49]. On this basis, this circuit has then been implemented on a robot, and it was shown that indeed it permitted to generate robustly the above mentioned variety of behaviors [63]. Recent further robotic models of both sensorimotor integration mechanisms for navigation and physiological implementation can be found in [17], [54], [55], and [94].

The Hydrodynamics of Swimming: Another example of the way robots have permitted the evaluation and elaboration of behavioral hypotheses is the RoboTuna project [149], which focuses on swimming mechanisms in fishes and dolphins. Indeed, the swimming performances of these animals are paradoxical: in 1936, zoologist James Gray calculated the muscular force that a dolphin would need to reach a speed of 20 knots, as sometimes observed, taking into account the resistance of water along the dolphin's body. The comparison of this result with the muscular models that had been established by dolphin biologists revealed astonishing: dolphins were seven times too weak.

A first hypothesis was that existing muscular models were false, and that their muscles would be much more efficient than those of terrestrial mammals. Yet, several researchers wondered whether the solution might lie in hydrodynamics. Indeed, in addition to their phenomenal speed, dolphins and fish are able to change suddenly and importantly their directions without loosing speed, which human-built water vehicles (boats, submarines, ...) built on classical hydrodynamics theory are totally incapable of, whatever their motor power. The "vortex hypothesis" was then put forward: while they are swimming, fish and dolphins could create unconventional hydrodynamic turbulences which they could leverage to decrease their trail and increase their power. Sixty years after Gray stated this paradox, the controversy was still unresolved since knowledge in biology and hydrodynamics did not advance far enough.

This is the context in which the Robotuna was set up (see http://web.mit.edu/towtank/www/). A team of engineers built a robot which morphology reproduced that of the tuna, equipped with a set of classical motors of which one did know precisely the power, and that allowed it to produce oscillations over the whole body, from the nose to the tail, such that it could swim in straight line in a basin. The parameters of these oscillations were then experimentally optimized to maximize the speed of the robot. This maximal speed was then compared to the speed predicted by the same calculus that James Gray used for the dolphin, and based on the one hand, on the perfect knowledge of the shape and motors of the robot, and on the other hand, on conventional hydrodynamics models: the experimental speed of the robot was significantly higher than the predicted speed (yet inferior to the speed of a real tuna). Gray's paradox had been reproduced, and this experiment allowed to render the vortex hypothesis much more plausible, while suggesting that the difference between nautical/terrestrial animal muscles was probably not the answer.

A number of more recent related projects investigating the use of robots to understand better swimming behavior in aquatic animals have flourished, among which [1], [14], [86], [91], [92], and [146]. In particular, [83] present, through the example of biorobotic investigations of the function of pectoral fins and of the hydrodynamic interactions between dorsal and caudal fins, a detailed argumentation of the impact that the use of robots can have in our scientific understanding of animal swimming:

Comparative approaches that examine locomotor function in different species, while invaluable, are limited by 
the investigator's inability to control for the many nonlocomotor differences among these species. Furthermore, it is difficult to alter the natural motions of the body and appendages in freely swimming animals to examine the effect of novel movement patterns on locomotor performances. And, robotic models can have their structure and material properties [...] altered at will, allowing a controlled investigation [...]. In our view, the marriage of robotic models with experimental analyses of biological locomotion promises to drive the next set of major advances in our understanding of aquatic propulsion [83].

\section{RoBOtS AS PROOF OF CONCEPTS AND EXPLORATION TOOLS}

Many robots, such as in the examples we just presented, have been constructed to study specific hypothesis on the behavior of relatively simple animals (for a larger panorama, see [161]). Yet, an equivalent number of robots have been built to explore and evaluate more general hypotheses, in particular in respect to the dynamic brain-body-environment relations or to the explanation of high-level cognitive phenomena such as imitation or language. We will now focus on such kinds of robot experiments.

The Role of the Body in Intelligent Behavior: During most of the 20th century, intelligence in high level organisms, such as humans, has been conceived as primarily consisting in information processing and symbol manipulation through rules of logical inference, in which the body is only an interface which transforms sensori inputs into internal symbols and executes commands computed by the inference system [28], [44], [108], [126]. This is sometimes referred as cognitivism, and is the theoretical foundation underlying both artificial intelligence and a large part of cognitive sciences [13], [97]. This approach has been extremely popular in the years 1950-1980, and reinforced by the first results of artificial intelligence, such as programs which could play checkers at a high level [130] or automatically prove theorems [50] - activities that cognitivism considered as the hallmark of intelligence.

Yet, the limits of this approach appeared rapidly: programs that were expert checkers player were incapable to learn how to play another game, and automatic theorem provers were not able to understand the simplest jokes [47]. The idea that cognitivism was bypassing fundamental principles of intelligence and behavior was beginning to make its way in the head of certain researchers.

A fundamental idea that was proposed was lying in the brain and a system of symbolic inference, intelligence, and behavior might be the result of the dynamical interactions between the brain, the body, and the environment. With this view, the body was not simply an interface, but fully part of mechanisms that gave rise to intelligence. Initially explored and argued by philosophers (e.g., [29], [46], [102], [103], and [153]), this hypothesis began to be naturalized, and thus, had its impact in cognitive sciences leveraged, only after a number of robotic experiments were able to articulate it and illustrate it explicitly and precisely [27], [87], [117]-[119]. We will now present two emblematic examples of such experiments.

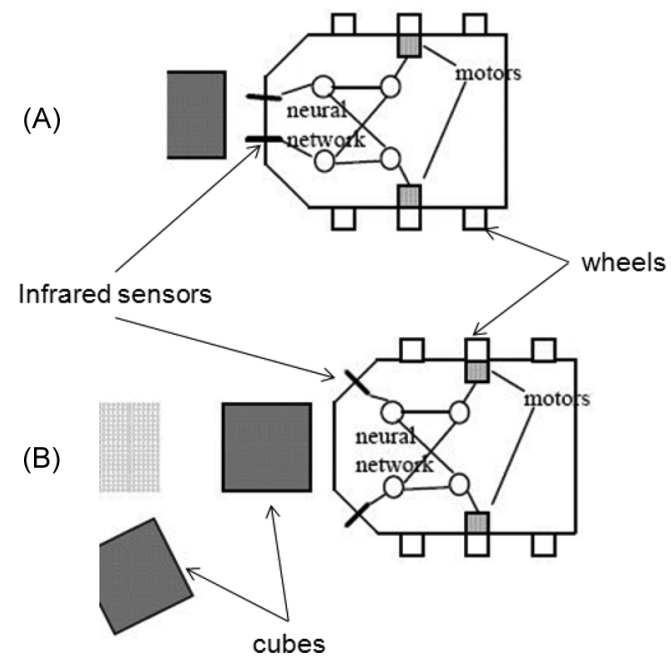

Fig. 1. Morphology of two types of Didabots (Adapted from Pfeifer, 2000).

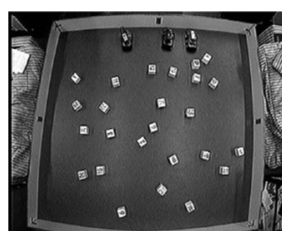

début
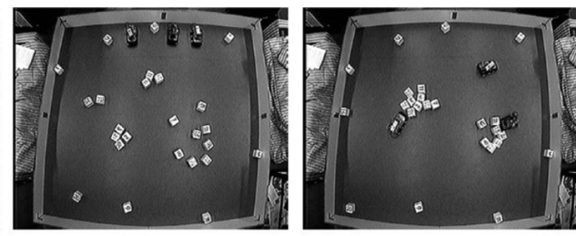

fin
Fig. 2. With morphology (B) and the same controller, cubes are clustered by Didabots. Adapted from [95].

The Didabots experiment [95] shows clearly the impact that morphology, in particular the physical and topological properties of the body and its sensors and motors, can have on behavior by comparing robot whose control system is the same, but the body is different. Didabots are wheeled robots with two infrared sensors that allow them to detect the presence of potential obstacle within a five centimetre range. Two versions of the Didabots were built, only differing by the exact positioning of these two sensors (see Fig. 1): in version (A), both sensors are positioned on the robot's "nose," whereas in version (B), both sensors are positioned on its "cheeks." The same controller is associated with both robots, corresponding to a mechanism for obstacle avoidance: if the sensor on the right is activated, then the motors are commanded to turn on the left; if the sensor on the left is activated, then the motors are commanded to turn on the right. This control system is quasi-identical to that of the "electric dog," except that the connections between left/right sensors and motors are reversed.

Then one puts two population of such robots in two identical arenas, one arena containing the first kind of Didabot, and the other arena containing the other kind. Also, light movable cubes are scattered in each arena. For robots with morphology (A), one observes what one could expect: they avoid obstacles. Robots (B) also avoid obstacles, but an additional phenomenon appears: after a certain amount of time, one observes a massive clustering of cubes (see Fig. 2). Thus, robots (B) have produced a behavior that external observers would qualify as a "cleaning" or "tidying up." As a matter of fact, this is explained by the fact that if a robot (B) approaches a cube right in front of it, it does 
not detect it as an obstacle since its sensors are slightly oriented on the sides (see Fig. 1). So, it pushes it until one of its sensors (left or right) perceives an obstacle, typically another cube, and begins to turn. The result is that two cubes that were initially noncontiguous end up next to each other. Step by step, all cubes are gathered into clusters in the arena. In conclusion, this experiment shows clearly that the behavior of robots (B) is neither the simple result of its brain/controller, nor the simple result of its body (which would not move without a brain), but a result of the interaction between the two.

In spite of the fact that this kind of experiment is extremely simple, it has an important scientific value since it allows researchers to explore something that is not possible with animals or humans, i.e., the comparison of behaviors resulting from different bodies with the same controller/brain (and vice versa). Indeed, even for a given species, natural variability among individuals makes it impossible to have two animals with exactly the same body or the same brain (even for true twins, because of epigenetic variation and stochasticity). There is no such experimental invariances in nature. It is even more problematic to imagine being able to make an experiment in which one would try to have the body of a lion controlled by the brain of a snake. Yet, this kind of experiment would allow us to advance considerably in our understanding of the relationships between the body and the brain in behavior. This shows the potentially important impact of robotic experiments in which for the first time one can consider the body as a variable [71].

The underlying idea of the Didabot experiment, that was systematically explored by Rolf Pfeifer and his colleagues [117]-[119], is that implicitly the body itself, through it physico-dynamic properties, is realizing a computation, called "morphological computation," which is as central to behavior as the computation explicitly realized by the brain. This is why for a given task an adequate morphology can allow the controller to be radically simplified.

A robotic experiment which permits to illustrate this point in an extreme manner was made by Tad McGeer with passive dynamic walkers [30], [99], [100]. Indeed, biped walking is an extremely complex behavior that puts into play the dynamic coordination of a large number of muscles, for which control systems of great complexity have been proposed to keep the body in equilibrium in spite of its inertia, of friction among its components, and of gravity (see for example the computational system that controls the walking of the ASIMO robot, [61]). In contrast with this approach, McGeer and other researchers elaborated an entirely mechanical two-legged device reproducing certain aspects of human legs morphology (see Fig. 3), with no controller at all (the device was only composed of metal and wood parts). He showed that with adequate distribution of weights, sizes, and positions in the different parts of this "robot's" body, and when put on top of a slightly descending slope, the robot would walk down keeping its dynamical equilibrium with movements that were astonishingly similar to human movements. Thus, the robot used gravity and dynamical mechanical properties of its body to move itself and keep its equilibrium without a brain (and without an internal source of energy). This seminal work participated in the launching of many subsequent projects dedicated at understanding how a well-designed morphology could

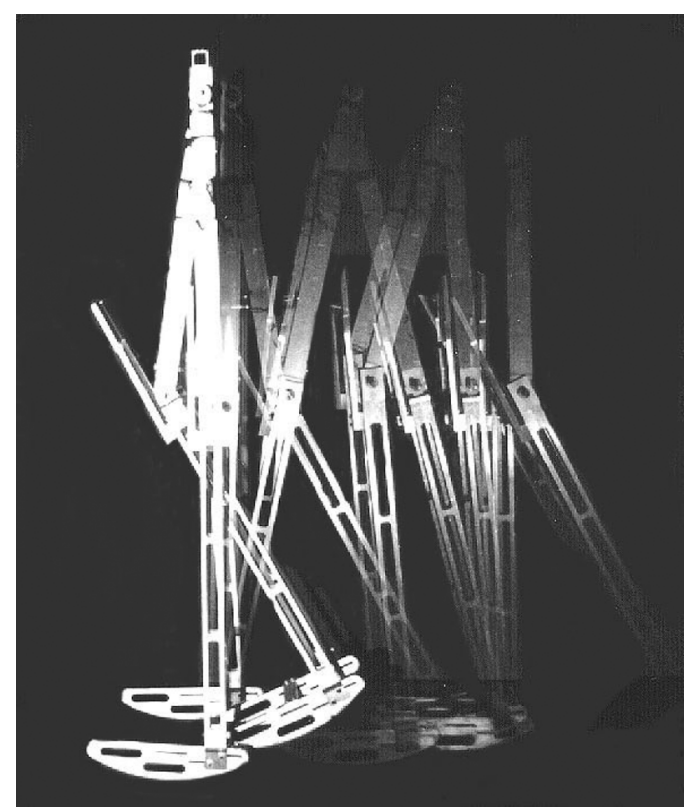

Fig. 3. A passive dynamic walker. Photograph from: http://ruina.tam.cornell. edu/hplab/pdw.html.

allow for both human/animal-style and human/animal-efficient walking. This includes the recent developments described in [32] where it was shown that adding simple controllers and actuators can allow a robot to walk on a flat ground with a limited consumption of energy, or in [56], [152] where further experiments showed that models based on compliant legs explained better the patterns of human walking that stiff-limbed passive dynamic walker models.

Furthermore, biorobotic investigations of the dynamics of the brain-body-environment coupling has also been conducted to study locomotion in nonhuman animals (e.g., lamprey, lobster, or salamander), such as described in the literature on central pattern generators for locomotion control. [67] presents a review on this specific topic and arguments precisely that the impact of biological sciences in robotics is accompanied by a symmetric impact of robotics in biological and behavioral sciences. This is illustrated by the lobster robot project [11], the lamprey robot projects [144], [165] or the Polichaete-like undulatory robot [150].

Intelligence Without Representation: One of the central concepts of cognitivism is representation, and in particular internal world models used by the brain to reason and deduce actions that should be undertaken to reach given goals, without which high level behavior is argued to be impossible [97], [108]. A whole series of robotic experiments came to put this stance into question [25]. A number of experiments showed how mobile robots could learn to achieve certain tasks in unknown environments, such as going back to a reference point or vacuum cleaning a space without using maps and only based on simple reflex mechanisms [5], [93]. Yet, more striking experiments dealing with cognitive capacities such as imitation have been achieved recently. Human babies begin to imitate their conspecifics very early in life, and many researchers have taken this fact as a proof that they may be endowed with 
advanced innate representations of their bodily self and its differences and correspondences with the body of others [60], [162]. Indeed imitation, which partly consists in reproducing an observed gesture, seems to imply the capacity for self- and other-awareness (the observed gesture is not one's own) and perspective taking. Yet, a robotic experiment presented in [2] shows that this conclusion cannot be done. In this experiment, the motor system of the robot consists in a arm, and it sensori system consists in a camera that can monitor movements in the image. The robot is endowed with a learning mechanism which allows it to learn to predict the visual movements that it perceives, and that are typically provoked by its arm passing in its field of view, in function of its motor commands. The robot is also equipped with a motivation system that implements the principle of cognitive homeostasis [96], [155]: when the robot detects an error between what it is predicting and what it is actually perceiving visually, it acts in order to restore the agreement between the positions/movements of its motors, perceived in a proprioceptive manner, and its visual percepts. Reference [2] showed that if one installed a second robot with the same morphology in front of this robot, and if this second robot was moving its arm, then the first robot spontaneously imitates those arm movements. Indeed, the perception of the movements of the arm of the second robot provoked a discrepancy between what the first robot predicted from its own movements. In order to have this prediction error disappear, the first robot then produces the same movement, visually covering the arm of the other robot. From an external observer's point of view, the robot imitates the gestures of the one in front of him. Yet, the knowledge of its control system allows us to state that it did not possess any kind of representation of the difference between what was relative to its own body and what was not (e.g., the body of another robot). Also, the robot did not possess a representation of its alter ego, and no mechanism for perspective taking was present. This experiment does not show that this mechanism corresponds to what happens in real human infants, and furthermore convincing further arguments show that more complex kinds of imitation observed in older children necessitate representations of the self and intentional understanding of the other [148]. Nevertheless, this experiment shows that on cannot deduce from the observation of early infant imitation that there necessarily is a representation of the self, of the other, and of the correspondences between the two.

Bootstrapping Foundational Representations of the Body and of the Self/Other Distinction: Emergentism/enactivism strongly challenged cognitivism/representationalism and showed that prespecifying representations of the world in an organism posed serious problem of scalability and adaptivity for this organism [25], [153]. Yet, it is also clear that human children's and adult's brains use sophisticated representations of their body and of their environment to achieve complex tasks [35], [81], [142]. This lead a number of researchers, especially in developmental robotics [10], [87], to identify the central question of how representations of the world can be formed and developed by an organism. At the same time, addressing this question can be viewed as an attempt to reconcile and bridge emergentism and cognitivism as argued by Luc Steels in his article "Intelligence with representation" [142], echoing Brooks' "Intelligence without representation" [25] 12 years later.

Among the most fundamental representations to be formed by a developing organism are those related to the self/other distinction as well as to the body schema [80], [127]. How can an agent, human, or robot, come to discover in the fuzzy-blooming sensorimotor flow some invariant structures corresponding to "self," "physical objects," and "other like-me"? How can a human or robot discover that it has two hands, two legs, and a head connected in a tree-like manner? Several robotic experiments have begun to propose stimulating answers in the last decade. First, several lines of work [74], [76], [88], [116], coming from different backgrounds, have converged to the idea that organisms could explore their sensorimotor loop and identify through motor babbling how much aspects of their sensory flow could be controlled by their own actions. This allows the organism to build categories on top of this continuous flow and based on the level of controllability: experiments have shown that robots could in that way form categories corresponding to the "self" (most controllable), to "physical objects" (partially controllable), and to "other agents" (least controllable). The robustness of this idea is shown by the fact that it can work with various different architectures for measuring controllability. For example, [74] have proposed an approach based on information theory, where controllability was measured as the mutual information between motor channels and aspects of the sensory flow. [72], [113], and [116] proposed a different architecture based on the concept of intrinsic motivation [41], [115] and in which the sensorimotor flow was actively and incrementally splitted and categorized according to a measure of learning progress, i.e., how much the robot's predictions of the consequences of its actions improve in a given subregion of the sensorimotor space, hence a measure of controllability. Furthermore, in this later approach, the discovery of the "self," "physical object," and "other" categories was achieved as a side effect of a general mechanism for spontaneous and active exploration of the sensorimotor space. Interestingly, it was shown that not only these categories were discovered and associated discrete representations were built, but that basic imitation and communication behaviors self-organized and developed on top of these representations, paving the way for higher level learning through social interactions [72], [113].

Once the "self" is discovered, remains the question of how an organism could discover, learn and represent its own body schema, i.e., an internal body map of the topology and/or structure of its body. Body maps are topological models of the relationships among body sensors and effectors, which human children learn progressively, abstract, and build upon to learn higher-level skills involving the relationships between the shape of the body and the physical environment [57]. Accordingly, inferring and reusing body maps from initially uninterpreted sensors and effectors has been identified as an important objective in developmental robotics [80]. Reference [121] presented 
a method, based on the use of distances such as the Hamming distance between uninterpreted sensors (i.e., sensors of which semantics and position are unknown), that allowed to reconstruct aspects of the relative positioning of the sensors of a robot when the physical structure of the robot is inarticulated (e.g., no arms or head that can modify the relative positioning of sensors). Reference [109] argued that sensors and motors could be conceptualized as information sources, and that tools form information theory could be used, such as the Crutchfield distance between two information channels, which is a metric [34]. Reference [109] showed that this distance, coupled with techniques for dimensionality reduction (e.g., multidimensional scaling), allowed to infer the topology of sensors and motors in an AIBO robot. Reference [70] showed that the topological structures inferred by this technique, called informational topological configurations, actually reflected the informational topological relationship between the structure of the body map, the structure of the motor activity, and the structure of the environment, in such a way that it can be used to represent the activity the robot is engaged in. Reference [133] showed how reconfigurable informational body maps in articulated robots could be learnt and dynamically predicted and generated, as well as reused to control the body and achieve tasks, such as tracking external stimuli. Hence, this series of experiments has allowed researchers to show that it was in principle possible for an organism to discover, learn, and build autonomously representations of its body map and reuse those representations to achieve tasks in the real world. As a consequence, this shows that innate dedicated representations of some fundamental aspects of cognition do not necessarily need to be prewired in the innate specification of an organism, as proposed by many cognitivist approaches [53], [122].

Self-Organization and Language Formation: Self-organization is a concept coming from the sciences of complexity which has had and is continuing to have deep consequences on the way scientists understand matter, life, and the mind. Self-organization characterizes systems in which macroscopic properties result from the microscopic properties, but are qualitatively different. For example, it characterizes the spontaneous formation of ice crystals in certain conditions of temperature and pressure, with branches which complex symmetric structures do not appear at the scale of water molecule that compose them (see Fig. 4). After several precursors like D'Arcy Thompson [147] who studied self-organization before the term was introduced, the concept appeared and really developed under the impulse of physicists and mathematicians like William Ross Ashby, Heinz von Foerster, Ilya Prigogine, or René Thom. It is now centrally involved in explanatory theories of many physical phenomena [12], including the formation of crystals, distribution of sizes of avalanches in sand piles or in mountains, dunes in the desert, river deltas, galaxies, or bubble polyhedrons at the bottom of water cascades. This has also begun to be used to explain living forms such as the formation of spots and stripes on the skin of animals, spiral or star patterns in bacteria colonies, the construction of termite nests or the formation of dynamically reconfigured social structures for hunting, or foraging in bees [26].

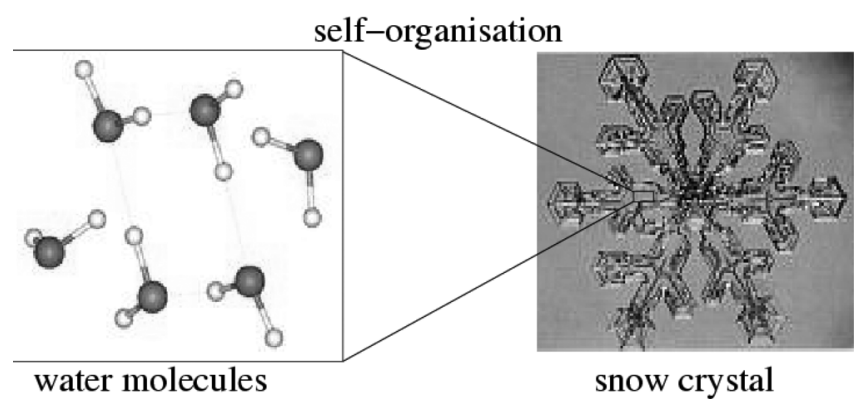

Fig. 4. Self-organization characterizes systems in which macroscopic patterns are qualitatively different from microscopic patterns, but result from them. For example, the symmetries and structures of branches in ice crystals are qualitatively different from the symmetries and structures of water molecules. Self-organization is observed both in the inorganic and organic worlds: this shows that the structures and forms of the living cannot be explained only by natural selection, but also need to involve the spontaneous self-organizing properties of the complex physical systems that compose and embed them.

Furthermore, these self-organized phenomena are often difficult to understand or predict intuitively and to formulate verbally. Mathematical modelling can be helpful, but as soon as interactions become too complex, analytical studies become intractable. One of the most efficient way to develop our understanding of the dynamics of self-organized complex systems is the use of computer simulations and robotic experiments. Indeed, they permit to elaborate models of which one knows all the assumptions, to run them, and then to observe the behavior varying in function of the values of the parameters of these models.

These computational models have been crucial in the work of physicists and biologists studying complex systems since the middle of the 20th century, starting from the work of Alan Turing on morphogenesis [151], or the work of Enrico Fermi on nonlinear dynamical atomic interactions. Since the beginning of the 1980's, they have been intensively used by ethologists and have allowed them to achieve significant advances in the understanding of insect societies [26].

More recently, several groups of researchers have proposed that self-organization might not only be crucial for understanding basic biological mechanisms, but also for understanding human cognitive and social mechanisms, in particular language origins [64], [85], [112], [138]. Thus, in the last two decades of the 20th century, it was proposed that certain linguistic structures, rather than being innately and explicitly encoded in the genome [123], could be the result of dynamical complex interactions between generic cerebral circuits, the auditory-gestural-phonatory apparati of individuals, individuals themselves and the function of communication [65], [141], [112]. Even further, it was proposed that languages themselves, conventional systems shared by all individuals in the same linguistic community which formation mechanisms have long remained mysterious, could be conceptualized as self-organized macrostructures resulting from cultural peer-to-peer interactions among individuals [138], [141]. Nevertheless, these hypotheses were audacious for two reasons: 1) they conflicted importantly with innatist Chomskian linguistics 
which were mainstream since the $1950 \mathrm{~s}^{1}$ 2) self-organized phenomena are complex and nonintuitive, implying that any verbal theory recruiting this concept is quite speculative. This is why, similarly to computer simulations and robotic experiments about social insects in ethology, robotic experiments have been elaborated to allow us to evaluate the coherence and the plausibility of these hypotheses. The use of robots is here essential, since hypotheses based on self-organization relied on the interactions between the brain, motor and perceptual apparati, and the physical and social environment. We will now present an experiment that permit to illustrate how robots can impact these fundamental questions traditionally formulated in human sciences.

The Origins of Speech Sounds and of the Associated Universals: In spite of the fact that humans are equipped with a phonatory and auditory system that could allow them to produce and perceive several hundreds of vowels and consonants, humans use most often only between five and ten vowels and only a few dozens consonants in a given language [134]. Furthermore, each language has its own system of vowels and consonants, resulting in a wide diversity across languages, but at the same time certain vowels and consonants are much more frequent than others: there are statistical structural regularities in human vocalisation systems, called universals [134]. Several reductionist explanations have been proposed, either based on arguments about morphology (e.g., Stevens [145]) or genetic (e.g., Mehler et al. [101]), but they did not provide an account of the duality diversity/universals that is observed. Certain researchers then proposed that human sound systems were optimal compromises between perceptual distinctivity and articulatory effort, and elaborated computer simulations that permitted to show that optimal vowel systems were indeed those that were most frequently observed in human languages [84]. Yet, these simulations were based on explicit optimization mechanisms and did not explain how this optimization might have occurred in nature. Furthermore, they did not allow us to understand how a given community of individuals could "choose" collectively one of the optimal systems rather than another.

Potential answers to these questions have been provided and evaluated in robotic models presented successively in [16], [19], and [110]-[112]. In these experiments, robots simulated in virtual worlds were equipped with models of the vocal tract, of the ear and the cochlea, and of neuronal circuits connecting these audito-phonatory systems (see Fig. 5). These robots interacted in a peer-to-peer manner and locally in space, through an imitation game: in an interaction, one of two robots chose a vocalization in its repertoire, produced it, and the second robot tried to imitate it by producing the vocalization of its repertoire

\footnotetext{
${ }^{1}$ What I call here innatist Chomskian linguistics refers to the part of linguistic theory which is structuralist and innatist, proposing that human brains possess innately language-specific structures such as an innate knowledge of possible syntactic structures in human languages, and which evolution is the result of a selection pressure for linguistic communication. Nowadays, the emblematic representation of this scientific trend is the argument proposed by Steven Pinker. On the contrary, Noam Chomsky is now rather an opponent of this approach, and is either neutral about the way syntactic structures are encoded in the brain and about the questions of the origins of such structures, or has proposed arguments defending the idea that the evolution of these structures could be a collateral effect, potentially self-organized, of biological evolution unrelated to communication. In this sense, Chomsky is not (or is not anymore) Chomskian.
}

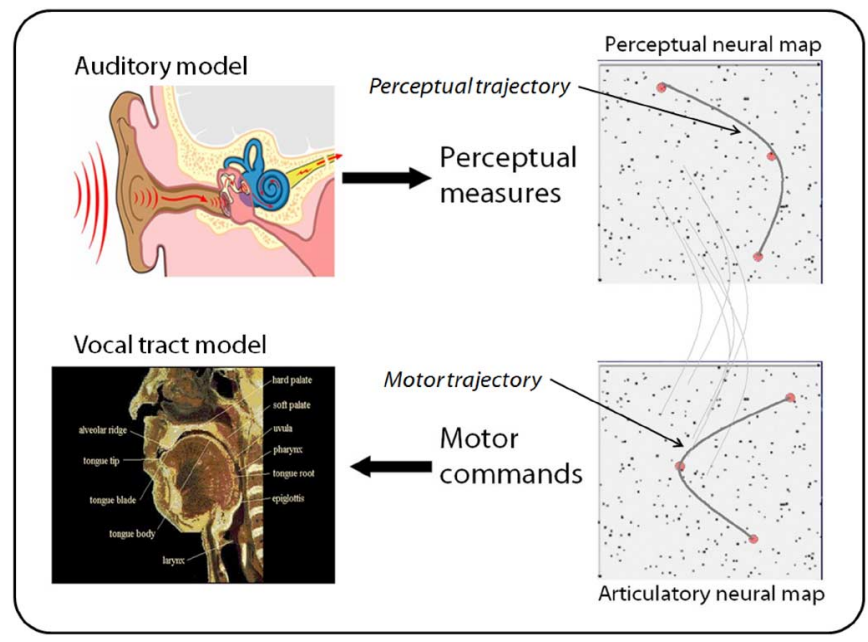

Fig. 5. The architecture of vocalizing robots presented in [112]. Virtual robots are equipped with an artificial auditory and phonatory system, coupled with a simple neural architectures composed of a perceptual and a motor neural map. Robots constantly babble, which allows them to learn the auditory-motor correspondences, but also impacts the babbling of their neighbors-plasticity in the neural system pushes robots to produce vocalizations which approximate the distribution of surrounding vocalizations. This phenomenon is known as phonological attunement. While this architecture can be used to show how a single babbling robot can learn the vocalizations of an existing combinatorial speech system, putting several babbling robots in a shared environment and without a preexisting speech system produces an interesting result-a combinatorial system of vocalization spontaneously forms and is shared by all individuals of the community. If one runs the experiment several times, one obtains different self-organized vocalization systems. Yet, statistical structural regularities appear over many simulations: we observe the duality universals/diversity.

that matched maximally. Then, the first agent checked whether the imitated sound was closer to the sound he produced initially than to other sounds in its repertoire. Then, it gave a feedback to the other agent about the success or failure of the imitation. In all cases, both agents updated their sound repertoires by building hypotheses to maximize successful imitation games in the future.

The sounds in the repertoires of agents consisted in associations between motor trajectories and acoustic trajectories, tagged with a score. The score of an association increased if it was used in a successful imitation, and decreased in the opposite case. Initially, agents began with an empty repertoire, which increased both through random inventions and through learning when they interacted with each other. The system of scores associated to sounds in the repertoires, coupled with the imitation game, introduced a cultural Darwinian dynamics in which sounds and groups of sounds could compete or cooperate to reproduce from brain to brains [114]. Experiments showed that rather quickly, a system of shared vocalizations self-organized in each community of robots, and this system was different in different communities. This allowed us to show how a conventionalized vocalization system could be formed in a community of individuals without central control. Furthermore, these experiments have shown that the vowel systems formed by communities of robots were at the same time diverse and characterized by strong statistical structural regularities: several vowel systems appeared significantly more frequently than others, yet sometimes rare systems were self-organized. More 
precisely, the most frequent vowel systems in robot communities were the same as the most frequent systems in human languages [112]. Thus, these simulations showed convincingly the plausibility and the coherence of the hypothesis of self-organization: simple mechanisms, through dynamic nonlinear interactions, could give rise to sound systems which fundamental characteristics matched those of human vocalizations.

Origins of Lexicon, Syntax, and Learnability: Together with the robotic experiments presented in previous paragraphs, a whole family of other experiments has come to reinforce the hypothesis of the self-organized origins of languages in recent years. For example, the Talking Head Experiments [69], [140], [141] has shown how a community of robots could build a shared lexicon only through local social interactions. This has allowed the experimenters to show that it was possible without assuming innate semantic categories, as it was proposed by a number of innatist theories [123]. Other experiments address the origins of syntactic and grammatical conventions (e.g., Kirby [75], Steels [143]), and showed how a linguistic system could evolve culturally to adapt to the cognitive biases of individuals (e.g., [166], [110], and [114]). In this context, they showed that linguistic systems could be selected for their learnability: certain structures evolved in such a way to become more easily learnable by individuals, under the generic constraints of their brain. Thus, these experiments have permitted to contradict the conclusions made by innatists from the observation that children learn their mother tongue so fast with only poor cues and stimuli [58], [121], [123]: they proposed that their brain had to adapt biologically to encode language specific contraints allowing individuals to learn language so smoothly. On the contrary, these robotic experiments showed that it was sensible to think that languages themselves could also have evolved in order to become easily learnable by pre-existing generic brains. These two hypotheses are by the way compatible and their combination is the topic of several current research projects.

\section{Robots AS SCIENTIFIC AND THERAPEUTIC TOOLS FOR ANIMAL AND HUMAN COGNITION}

At the beginning of the 20th century, Hammond and Miessner's robot, and a few decades later the machines of Ashby and Walter, indirectly provided the opportunity to highlight the way humans could overinterpret the behavior of simple entities. Thus, they were not only useful in the debate opposing vitalists and materialists, but also permitted to study human psychology. In the last decade of the 20th century, several researchers have begun to employ voluntarily robots as tools for direct experiment and study of animal and human behavior, as opposed to using them as models of these behaviors as those presented in previous sections. Yet, a similarity with modelling work is the use of robots in the context of experiments addressing a large spectrum of behaviors: we will give the example of studies of certain behaviors in social insects, then in mammals like dogs, and finally in humans.

Bee Dancing: The work presented in [104] on dances in honey bees is a paradigmatic example of how robots can be used to study directly the behavior of animals. Karl von Frisch defended the theory that bees used their dances as partially arbitrary symbols to transmit the position of nectar sources to their conspecifics [156]. According to this theory, when a bee comes back to the beehive to transmit information about the location of a nectar source, there are two alternatives. If this source is relatively close (less than one hundred meters), the bee performs a round dance, and other bees come to touch her antennas to smell the odor of the nectar still sticking on the hairs of the dancer. Then, they go to search for the nectar driven only by their sense of smell. On the contrary, if the nectar source is relatively far, the bee performs the "waggle dance". It consists in the successive path of two semicircles passing through a central straight line on which the bee shakes its abdomen and emits vibrations with its wings. von Frisch proposed that the speed of waggling and the frequency of the vibration, as well as the duration necessary to go around a semicircle, determined the distance to the nectar source, while the angle formed by the central line and the semicircle determined the direction of the nectar source in relation with the location of the sun. In spite of its Nobel prize in 1973, the theory of von Frisch was vividly criticized by several researchers that claimed that even in the case of a far nectar source, they were still mainly guided by their sense of smell [163]. This controversy was on the one hand based on the fact that bees are equipped with a very sophisticated smell apparatus, and on the other hand on the relative uncertainty of the role of the various dance components in the theory of von Frisch. Indeed, because of the intrinsic variability of each dance and each bee, and because of the fact that a bee always produces concurrently a complex flow of signals which are difficult to untangle and which we are not sure to have all identified, the theory of von Frisch is difficult to accurately validate experimentally.

In 1992, an experiment based on the use of a robot came to confirm this theory. Michelsen and his colleagues developed a small robot of the same size and shape as a bee, linked to a computer and teleoperated by a computer program allowing the robot to perform dances in beehives and study their impact on real bees. In a first stage, some wax was put on the robot so that it would be impregnated with the smell of bees and would be recognized as a conspecific. Once the habituation stage was over, researchers could command the robot to perform a number of dances in which they could independently control the speed of waggling, the frequency of vibrations (produces thanks to the acoustic waves of small artificial wings), the duration used for going around semicircles and the angle between the central line and the two semicircles. The unique advantage of using a robot was on the one hand the possibility to repeat exactly the same dance many times without variability, permitting statistically significant observations of the reaction of bees, and on the other hand, to be able to remove certain components of the dance, control them one by one, and even to propose dances with contradictory components which are never generated by real bees, but that may highlight the way these cues are jointly used. Furthermore, the experimenter could ask the robot to make a dance indicating to the bees that there was a nectar source at a place where there was no nectar: this allowed him to avoid the possibility that bees could be guided by their sense of smell. The results of these experiments were very clear: the experimenter's robot managed to "send" the bees where the experimenters wanted to without smell cues. Moreover, because the use of a robot permitted to argue that the only signals transmitted 
to the bees where those implemented in the robot and corresponding to von Frisch's theory, the foundations of this theory were confirmed. Yet, the use of robots allowed the experimenter to go even further and refine von Frisch's theory: Michelsen and its colleagues showed that in fact, the essential part of the dance was the straight line, during which the bee shakes its abdomen and emits vibrations, whereas the semicircle, during which the bee stops to shake and to vibrate, did not seem to have the crucial role of direction indicator that von Frisch had proposed. On the contrary, it seemed that the combination of the waggling and the sound was the indicator of both the distance and the direction of the nectar source. This shows the fundamental role that the robot played in the understanding of bee dancing.

The Recognition of Conspecifics in Dogs: Another example of the use of robots as tools to perform experiments with animals is presented in [79], and concerns the study of conspecifics recognition, i.e., how dogs can recognize that other entities are (or are not) of the same "kind"/species. The study of mechanisms of social recognition, and in particular recognition of animals of the same species, is an important topic in ethology [31]. For numerous species, ethologists try to understand whether conspecifics recognition is innate or learned, how it is developing, whether it depends on the context, but also which are the visual, behavioral, acoustic, or olfactory cues which underlie this capacity. The problem is similar as the one we described for bees: in nature, the stimuli that animals perceive are always multidimensional and multimodal, mixing many potential cues that experimenters cannot control. To circumvent this problem, certain researchers tried to use artificially and systematically generated images to study the reaction of animals, such as fish [31], or elaborated mock ups of which they could control visual appearance and smell [81]. Yet, it was shown that the lack of movement of these stimuli could be an obstacle to the reaction of the animals under study, because they could quickly consider them as nonliving and thus, not pay attention to the other stimuli that they use to recognize their conspecifics [124].

This is the reason why Kubinyi and her colleagues, in the context of the study of conspecifics recognition in dogs, proposed to use an AIBO robot, which shape and size is similar to that of several dog species, and which programmed movements are inspired from dog behavioral models [7]. Thus, they set up systematic experiments comparing the reaction of dogs with various ages and sexes when they interacted with four types of partners: a young dog of the same size as the AIBO, a standard AIBO robot, an AIBO robot with a fur, and impregnated with dog smell, and a remote-controlled car. These interactions took place in two different situations: neutral interactions during which the dog and its partner are in the same room with humans, and interactions while the dog is feeding itself in a bowl of food and the partner is headed towards the bowl (the robot and the remote-controlled car are programmed to go toward the bowl). In each trial, experimenters monitored precisely the behavior of the dog under study, including the duration of approach or remoteness with respect to the partner, the duration of looking towards the partner, the number of times the dog came to smell the partner, and the number of times the dog barked or growled. Once these experiments were terminated, it was possible to show that the AIBO robot covered with a smelling fur was inspected by all dogs and in all situations as much as the real young dog, and significantly more than the AIBO robot without a fur and than the remote controlled car. They also showed an important difference among adult and young dogs when they were confronted to the partner while eating: most of young dogs growled towards the robot and the car when they approached their dish, while most of adult dogs ignored both. Finally, the comparison between the results of this experiment and previous experiments that used mock ups or photographs showed that the use of robots permitted to trigger a significantly higher number of reactions in dogs.

This shows several things. First, the use of robots allows researchers to make experiments which are at the same time more controllable that the use of natural partners (conspecifics or animals from another species) and more sophisticated than the use of photos or mock ups. Second, four-legged movements combined with an adequate smell draws the attention of dogs in a similar way as living beings (but not necessarily conspecifics). Finally, this experiment brings us hints that support the idea that conspecifics recognition is a capacity that develops in dogs: juveniles seem to have only a very approximate capability to identify which other entities are of the same "kind" as themselves. In conclusion, we see how the use of a robot can allow us to improve the way we understand how dogs distinguish the animate from the unanimate, and how they distinguish other dogs from other animals.

Robots and Autism in Human Children: Robots are not only used as experimental set ups to study the behavior of insects or mammals, but also for the study of humans. In particular, recent years have witnessed the development of several research groups putting forward the interest of using robots to study, diagnose, and even contribute to the therapy of children with autism disorders [38], [45], [77], [132], [137]. Autism refers to a family of problems in child development characterized by a number of symptoms [154]: 1) social interaction deficit: autistic children focus much more their attention on objects than on the persons that are around them, they are not socially proactive, and they have many difficulties to interpret the behavior of others; 2) communication problems: they often have language disorders, which can be observed through wrong choices of intonations or words for example; 3 ) stereotypic behaviors and an aversion for novelty: autistic children tend to retreat into rituals and fear situations which deviate from their routine.

Diagnosing Autism: One of the first obstacles faced by scientists and physicians is the diagnosis [132]. Indeed, autism covers a large spectrum of developmental disorders and its specification is behavioral [154]: there is neither blood nor genetic test, and no clear markers that can be seen with brain imagery. This can be explained by the fact that this spectrum of disorders is caused by a varied set of mechanisms that are mostly unidentified by the scientific community. The gathering of reliable and statistically significant data about populations of autistic children is an essential step to progress in our understanding of their deficits. This requires diagnosis and evaluation protocols that are as precise and as objective as possible. Unfortunately, even if standardized protocols exist (e.g., [106]), they rely on the intuitive evaluation of clinicians about the social capacities of the child, and relative to the way they control their gaze, to their fa- 
cial expressions, and to their gestures. Thus, one can often observe disagreements among clinicians, which are exacerbated by the fact that autistic children typically refuse to interact with them.

Scassellati proposed to experiment the use of robots to improve the quality of diagnosis, as well as the monitoring of the evolution of social and cognitive capacities in autistic children [132]. This idea relies on an observation made by all the research groups interested in this topic: autistic children are highly motivated by interaction with robots, more than with traditional toys, and even the children that refuse to interact with clinicians or therapists engage in social interaction with the robot when they are present [38], [132], [137]. Moreover, it is possible to program robots so that they become interactive and produce stimuli that are really standardized and repeatable, allowing clinicians to use the same test for all children. This can be coupled with the use of a set of methods for recording and analyzing social situations elaborated by the social robotics community (e.g., Breazeal, Robinson-Mosher, and Scassellati [129]), permitting to measure precisely in real-time elements such as gaze direction, position in the environment relative to persons and objects, or the characteristics of voice intonation, which are relevant cues for the diagnosis of autism. Furthermore, as these robots can have a simple shape and be easy to manufacture, it is possible to have them go out of hospitals and have children continue to play with them at home. If this would be realized at a large scale in a systematic manner, much more precise and natural data could be gathered about each child, which could allow clinicians to adapt and follow more efficiently the therapies. This would also allow for statistical data over these children populations, paving the way for advances in our understanding of this spectrum of developmental disorders [132].

Robots as Therapeutic Tools: In addition to the potential benefit of robots as tool for diagnosing and monitoring autism in children, several research groups also showed that they constituted a potential tool for therapy [38], [45], [77], [132] [137]. As explained earlier, robots provoke a high interest in autistic children: very often, they are lead into an interaction with robots that involves spontaneous gestural and vocal imitation, smiles, free play, and stop their stereotypic behaviors, which can be observed much more rarely when they interact with a human partner or with simpler toys [164]. One of the reasons is that these children have great difficulties to make sense of vocal, gestural and facial expressions in adults and other children because of their complexity and their variability. For example, in facial expressions, it seems that they do not manage to distinguish, among the many muscular cues of the face, those that are characteristic of one emotion and those that are not and result from the natural variability of the movements of faces. This lack of understanding provokes a retreat and a psychological blocking which increases their social know-how deficits as time passes. This is also one of the reason why they focus their activities on stereotyped activities that involve repetitive manipulation of physical objects or the generation of ritual gestures. In this landscape, robots appear to fill in the gap between physical objects and human partners. Indeed, because they are self-propelled, apparently autonomous, and often have anthropoid or animaloid shapes [37], [45], [78], [157], children easily consider them as animate beings with intentions [125]: robots are considered as potential social partner and draw children's attention. Yet, as opposed to human social partners, the behavior of robots can be simple and predictable, hence reassuring and allowing children to engage in positive interactions rather than to retreat from interaction with other humans.

First, this has the huge advantage to allow children to gain a little bit of confidence about their own social behavior [38]. Second, thanks to the fact that robots can be tuned-in shape and behavior - to every single child (this is the core of the European project IROMEC), the context of these positive interactions can be used by therapists to have children practice social skills that are specific to their developmental disorders, e.g. gestural imitation [37], facial imitation [107], turn-taking [39], bodily awareness [38], or joint attention [45]. Finally, a number of recent experiments showed that the use of robots with these children did not only foster the practice of fundamental social interactions between the child and a robot, but could also provoke unusually rich social interaction between the child and other humans present in the room with the robot. For example, [45] showed that the use of a robot could permit to establish an increased level of joint attention (visual contact, physical proximity) and imitations of smiles among children confronted together with the same robot. (Robins and [36]) showed that the use of a robot could allow the therapist to establish a much more positive relationship with the child. Thus, it seems that robots can be used as social mediators between autistic children and other humans, fostering a more efficient learning of social know-how and a better integration in our society.

\section{DISCUSSION}

One century after the pioneering "electric dog" of Hammond and Miessner, interactions between robotics and the sciences of the living, of the mind, and of behavior have flourished. In particular, in addition to the impact that biology can have on the way engineers conceive robots, which we have not dwelled on in this article since it is abundantly described in the literature (e.g., [4], [6], [10], [15], [36], and [52]), we have seen that the construction and use of robots could in itself transform the way we conceptualize the living.

As it was argued in this article, this is particularly true for behavioral and cognitive sciences. First, we have reviewed how the implementation of specific behavioral theories, following the example of the work of Hammond and Miessner, could allow researchers to evaluate their plausibility and their coherence, or on the contrary to invalidate them. We have seen how robotic experiments permitted to identify sufficient conditions, or prove the non-necessity of the presence of certain mechanisms, to account for certain behaviors and that this could have a crucial role in the structuration of the scientific debate. Even more than the evaluation of existing hypotheses, robotics research projects allow us to articulate and elaborate our scientific intuitions about novel concepts such as the role of morphology in adaptive behavior, the bootstrapping of foundational representations of the body and of the self/other disctinction, or the role of self-organization in language formation. We have shown that they can even be the source of novel hypotheses such as those related to Darwinian cultural dynamics in the evolution of languages and their impact 
on learnability. We have also argued that robots could reveal to be precious tools to elaborate experimental set ups for the direct and systematic study of the properties of animal behavior and human psychology. Finally, a number of recent works suggest that robots may be used as unique therapeutic tools to attempt to remedy human developmental disorders such as autism.

Yet, in spite of these many types and examples of contributions, it has to be recognized that the use of robots is still far from being considered as a standard tool in biological, behavioral, and cognitive sciences. Even more, it seems that many of the contributions of robotics to these sciences in the past have been forgotten. This is exemplified again by the "electric dog": after being centrally reused in biological debates about theories of autonomous insect phototaxis as well as massively publicized in the press at the beginning of the 20th century, it quickly became forgotten. Forty years later, in the late 1940s, researchers like Grey Walter [21], [159] rebuilt essentially the same phototaxic robots, which were again used as exploratory tools in neurophysiology (Walter was actually a neurophysiologist) to show that simple neural circuits could give rise to complex behaviors, and which were again the topic of wide media coverage, as if no one in the past had ever built such machines. Being part of the cybernetics movement, these robots also contributed to the technical foundations of control theory [66], a field in which cumulative work has since been ongoing. Yet, from the biological, behavioural and cognitive sciences point of view, the work of Grey Walter and his peers like W. Ross Ashby [9] again faded out and became mostly forgotten for a new period of forty years. In the middle of the 1980's, a new group of researchers, and in particular Braitenberg [22] and Brooks [24], began to build again variants of the "electric dog" and confronted them with current mainstream (cognitivist) theories of cognition, showing that complex behavior could be generated with direct sensorimotor coupling, and no or very little representation. The same scenario happened: in addition to wide media coverage, it triggered again novel profound debates in behavioral and cognitive sciences, ranging from neuroscience (Braitenberg was a neuroscientist) to philosophy (e.g., Dennett discussed extensively the epistemic impact of Brooks' robots, see Dennett [42]).

How can we explain this scientific forgetfulness and relative inefficiency to achieve cumulative scientific progress in the use of robots in biological, behavioral, and cognitive sciences? First, there is probably one institutional factor: robotics and all these sciences are strong well-identified, but remote disciplines, implying that their actors live in often disconnected departments in universities, which centre of gravity evolves independently: has a quasi-mechanical consequence, bridges made over these scientific continents can hold only if there would be continuously a critical mass of researchers reshaping and repairing the bridge, which has not been the case in the past century.

A second reason is that trying to link psychological concepts with the behavior of machines has persistently been considered as a dubious activity by many researchers in both hard and human sciences, often due both to philosophical, cosmogonic or religious preconceptions [68], and to the excessive and deforming wide media coverage which often distorts the actual scientific work and in the end discredits it in the eye of their peers [21].
A third and more profound reason is probably linked to the very nature of robotics' contributions to behavioral and cognitive sciences. First of all, we can find a unifying feature of all the types of contributions we identified: in most cases, robots are mainly used as physical thought experiments which main impact consists in studying the internal (in-)coherence of theories and in inspiring new theories in behavioral and cognitive sciences (see [43] for a related discussion on the use of computational simulations in the artificial life field). Thus, the use of robots in this context can really technically be considered as a kind of experimental metaphysics, and thus is not directly science but an activity which stimulates science. This is actually a logical conclusion that can directly be made from many of the practical examples described in this article. Indeed, one of the most prominent contributions of robotics is to show how the physical and material properties of the body can be essential for understanding behavior and control in an organism. Robots allow for the first time in scientific enquiry to systematically explore this rather old idea, because they enable researchers to consider the body as an independent variable [71]. But at the same time, because precisely robots are robots and thus have a body which has different material and physical properties than any existing animal, even if inspired by animals, a corollary is that it is impossible to explain directly any particular behavioral property of an animal through a robotics experiment. The goal of metaphysics is to study constraints and principles over the world of possible and logical physics, but any particular physics (e.g., the behavior of a particular existing animal) requires to be studied by traditional sciences. And this probably explains why robotics contributions to behavioral and cognitive sciences tend to become forgotten in these sciences: while fundamental to entrain conceptual breakthroughs, any particular explanation of a property of animal behavior or cognition always has to end up by very traditional direct work and experiments on the real animal by biologists or psychologists. And scientific records tend to memorize only the end point and to forget the path that led to it, especially if it was long, distributed and scattered with populations of metaphysical debates.

A last reason which makes it even more difficult for robotics to become visible in the long-term for behavioral and cognitive sciences is the fact that in many works there is a tight entanglement between the scientific goal of understanding better nature and the engineering goal of building more efficient machines. There are many good reasons to actually target these two goals at the same time. But the consequence is also that in the eyes of outside and out-of-discipline scientific observers, the scientific message gets blurred and it is often difficult to extract what the feedback to behavioral and cognitive sciences actually is.

For these reasons, if one wants to remedy this relative forgetfulness and inefficiency in cumulative progress, there is a crucial need for historical and epistemological accounts studying how the use of robots has had and continues to have an impact on behavioral and cognitive sciences. As this is a complex multifaceted interdisciplinary endeavour, these accounts should be multiple, written by different authors such that different points of views can be confronted, and span the range from technical and detailed investigations targeted at specialists to general overviews targeted at a wide audience. This article 
was written as an attempt to address this challenge through a choice, presentation, and conceptualization of examples that in my opinion are emblematic and for which I have tried to separate out their contribution to behavioral and cognitive sciences from engineering goals. While a number of other attempts had already been made in the past, as explained in the introduction, I believe that as a whole many more will be needed to reach a strong and shared understanding of the impact of robotics in behavioral and cognitive sciences. History of science and epistemology are high priorities for durable and cumulative progress at the frontiers of these fields.

\section{REFERENCES}

[1] P. V. Alvarado and K. Youcef-Toumi, "Performance of machines with flexible bodies designed for biomimetic locomotion in liquid environments," in Proc. IEEE Int. Conf. Robot. Autom., Barcelona, Spain, 2005, Apr. 18-22, 2005, pp. 3324-3329.

[2] P. Andry, P. Gaussier, S. Moga, J. P. Banquet, and J. Nadel, "Learning and communication in imitation: An autonomous robot perspective," IEEE Trans. Syst., Man, Cybern., Part A: Syst. Humans, vol. 31, no. 5, pp. 431-444, Sep. 2001.

[3] M. A. Arbib, S. Amari, and P. H. Arbib, The Handbook of Brain Theory and Neural Networks. Cambridge, MA: MIT Press, 2002.

[4] M. Arbib, G. Metta, and P. van der Smagt, "Neurorobotics: From vision to action," in Springer Handbook or Robotics, B. Siciliano and O. Khatib, Eds. New York: Springer-Verlag, 2008, ch. 64.

[5] R. Arkin, "Behavior-based robot navigation in extended domains," $J$. Adapt. Behav., vol. 1, no. 2, pp. 201-225, 1992.

[6] R. Arkin, Behavior-Based Robotics. Cambridge, MA: MIT Press, 1998.

[7] R. C. Arkin, M. Fujita, T. Takagi, and R. Hasegawa, "Ethological modeling and architecture for an entertainment robot," in Proc. IEEE Int. Conf. Robot. Autom., Seoul, Korea, 2001, pp. 453-458.

[8] W. R. Ashby, "Adaptiveness and equilibrium," J. Mental Sci., vol. 86, pp. $478-483,1940$.

[9] W. R. Ashby, Design for a Brain. London, U.K.: Chapman and Hall, 1952.

[10] M. Asada, K. Hosoda, Y. Kuniyoshi, H. Ishiguro, T. Inui, Y. Yoshikawa, M. Ogino, and C. Yoshida, "Cognitive developmental robotics: A Survey," IEEE Trans. Autonom. Mental Develop., vol. 1, pp. 12-34, May 2009.

[11] J. Ayers and J. Witting, "Biomimetic approaches to the control of underwater walking machines," Philosoph. Trans.: Math., Phys., Eng. Sci. (Series A), vol. 365, no. 1850, pp. 273-295, 2007.

[12] P. Ball, The Self-Made Tapestry, Pattern Formation in Nature. Oxford, U.K.: Oxford Univ. Press, 2001.

[13] M. A. Boden, Mind as Machine: A History of Cognitive Science. Oxford, U.K.: Oxford Univ. Press, 2006.

[14] P. R. Bandyopadhyay, "Trends in biorobotic autonomous undersea vehicles," IEEE J. Ocean. Eng., vol. 30, no. 1, pp. 109-139, Jan. 2005.

[15] G. A. Bekey, Autonomous Robots: From Biological Inspiration to Implementation and Control. Cambridge, MA: MIT Press, 2005.

[16] A. Berrah, H. Glotin, R. Laboissière, P. Bessière, and L. J. Boë, "From form to formation of phonetic structures: An evolutionary computing perspective," in Proc. 13th Int. Conf. Evol. Comput. Mach. Learn., Bari, Italy, 1996, pp. 23-29.

[17] A. Beyeler, J.-C. Zufferey, and D. Floreano, "Vision-based control of near-obstacle flight," Autonom. Robots, vol. 27, no. 3, pp. 201-219, 2009.

[18] L. J. Boe, J. L. Schwartz, and N. Vallee, "The prediction of vowel systems perceptual contrast and stability," in Fundamentals of Speech Synthesis and Recognition, K. E. Editors, Ed. Chichester: Wiley, 1995, pp. 185-213.

[19] B. de Boer, The Origins of Vowel Systems. Cambridge, U.K.: Oxford Univ. Press, 2001.

[20] M. Boden, Mind as Machines: A History of Cognitive Science. Oxford, U.K.: Oxford Univ. Press, 2006.

[21] M. Boden, "Grey Walter's anticipatory tortoises," Rutherford J., vol. 2, 2007.

[22] V. Braitenberg, Vehicles: Experiments in Synthetic Psychology. Cambridge, MA: MIT Press, 1984.

[23] C. Breazeal, Designing Sociable Robots. Cambridge, MA: MIT Press, 2002.

[24] R. A. Brooks, "A robust layered control system for a mobile robot," IEEE J. Robot. Autom., vol. 2, no. 1, pp. 14-23, Mar. 1986.
[25] R. A. Brooks, "Intelligence without representation," Artif. Intell., vol. 47, pp. 139-160, 1991.

[26] S. Camazine, J.-L. Deneubourg, N. R. Franks, J. Sneyd, G. Theraulaz, and E. Bonabeau, Self-Organization in Biological Systems. Princeton, NJ: Princeton Univ. Press, 2001.

[27] H. Chiel and R. Beer, "The brain and body: Adaptive behavior emerges from interactions of nervous system, body and environment," Trends Neurosci., vol. 20, no. 12, pp. 553-557, 1997.

[28] N. Chomsky, Syntactic Structures. The Hague: Mouton, 1985, 1957, Reprint. Berlin and New York.

[29] A. Clark, Supersizing the Mind: Embodiment, Action, and Cognitive Extension. Oxford, U.K.: Oxford Univ. Press, 2008.

[30] M. Coleman and A. Ruina, "An uncontrolled toy that can walk but cannot stand still," Phys. Rev. Lett., vol. 80, no. 16, pp. 3658-3661, Apr. 1998.

[31] P. W. Colgan, Comparative Social Recognition. New York: Wiley, 1983.

[32] S. H. Collins, A. L. Ruina, R. Tedrake, and M. Wisse, "Efficient bipedal robots based on passive-dynamic Walkers," Science, vol. 307, pp. 1082-1085, 2005.

[33] R. Cordeschi, The Discovery of the Artificial: Behavior, Mind and Machines Before an Beyond Cybernetics. Norwell, MA: Kluwer, 2002.

[34] J. P. Crutchfield, "Information and its metric," in Nonlinear Structures in Physical Systems-Pattern Formation, Chaos, and Waves, L. Lam and H. C. Morris, Eds. Berlin, Germany: Springer-Verlag, 1990, pp. 119-130.

[35] W. A. Croft and A. Cruse, Cogn. Ling. Cambridge, U.K.: Cambridge Univ. Press, 2004.

[36] K. Dautenhahn, "Socially intelligent robots: Dimensions of humanrobot interaction," Phil. Trans. Roy. Soc. B: Biol. Sci., vol. 362, no. 1480, pp. 679-704, 2007.

[37] K. Dautenhahn and A. Billard, "Games children with autism can play with Robota, a humanoid robotic doll," in Proc. Cambridge Workshop Universal Access Assistive Tech., New York, NY, 2002, pp. 179-190, Springer-Verlag.

[38] K. Dautenhahn, I. Werry, T. Salter, and R. T. Boekhorst, "Towards adaptive autonomous robots in autism therapy: Varieties of interactions," in Proc. IEEE Int. Symp. Comput. Intell. Robot. Autom., Piscataway, NJ, 2003, pp. 577-582.

[39] K. Dautenhahn and I. Werry, "Towards interactive robots in autism therapy: Background, motivation and challenges," Pragmatics Cogn., vol. 12, no. 1, pp. 1-35, 2004.

[40] J. L. Davis and A. Rudolph, A Lamprey-Based Undulatory Vehicle. Cambridge, MA: MIT Press.

[41] E. Deci and R. Ryan, Intrinsic Motivation and Self-Determination in Human Behavior. New York: Plenum, 1985.

[42] D. Dennett, Brainchildren: Essays on Designing Mind. Cambridge, MA: MIT Press, 1998.

[43] E. A. Di Paolo, J. Noble, and S. Bullock, "Simulation models as opaque thought experiments," in Proc. 7th Int. Conf. Artif. Life, Cambridge, MA, 2000, pp. 497-506, MIT Press.

[44] J.-P. Dupuy, Aux Origines Des Sciences Cognitives. Paris, France: La Découverte, 2005.

[45] A. Duquette, F. Michaud, and H. Mercier, "Exploring the use of a mobile robot as an imitation agent with children with low-functioning autism," Autonom. Robot, vol. 24, pp. 147-157, 2008.

[46] H. Dreyfus and S. Dreyfus, Mind Over Machine. New York: Free Press, 1982.

[47] H. Dreyfus, What Computers Can't Do: A Critique of Artificial Reason. New York, NY: Harper and Row, 1972.

[48] H. Driesch, Philosophie des Organischen. Leipzig: Engellman, 1909.

[49] M. Egelhaaf, A. Borst, and W. Reichardt, "The nonlinear mechanism of direction selectivity in the fly motion detection system," Naturwissenschaften, vol. 76, pp. 32-35, 1989.

[50] G. W. Ernst and A. Newell, GPS: A case study on generality and problem solving. New York: Academic, 1969.

[51] S. Exner, The Physiology of the Compound Eyes of Insects and Crustaceans. Berlin, Germany: Springer-Verlag, 1891, pp. 130-131, Translated by R. C. Hardie.

[52] D. Floreano and C. Mattiussi, Bio-Inspired Artificial Intelligence: Theories, Methods and Technologies. Cambridge, MA: MIT Press, 2008.

[53] J. Fodor, LOT 2: The Language of Thought Revisited. Oxford, U.K.: Oxford Univ. Press, 2008.

[54] N. Franceschini, F. Ruffier, and J. Serres, "A bio-inspired flying robot sheds light on insect piloting abilities," Curr. Biol., 2007.

[55] S. N. Fry, N. Rohrseitz, A. D. Straw, and M. H. Dickinson, "Visual control of flight speed in Drosophila melanogaster," J. Experi. Biol., vol. 212, no. 8, pp. 1120-1130, 2009.

[56] H. Geyer, A. Seyfarth, and R. Blickhan, "Compliant leg behaviour explains basic dynamics of walking and running," Proc. R. Soc. B, vol. 273, pp. 2861-2867, 2006. 
[57] J. J. Gibson, 1977, “The theory of affordances," Perceiving, Acting, and Knowing R. Shaw and J. Bransford, Eds.

[58] E. Gold, "Language identification in the limit," Inform. Control, vol. 10, pp. 447-474, 1967.

[59] K. G. Götz and H. Wenking, "Visual control of locomotion in the walking fruitfly drosophila," J. Compar. Physiol., vol. 85, pp. 235-266, 1973.

[60] P. Guillaume, L'imitation chez l'enfant. Paris, France: Alcan, 1925.

[61] M. Hirose, Y. Haikawa, T. Takenaka, and K. Himi, "Development of humanoid robot ASIMO," in Proc. IEEE/RSJ Int. Conf. Intell. Robot. Syst., Workshop 2, 2001.

[62] O. Holland, "Grey Walter: The pioneer of real artificial life," in Proc. 5th Int. Workshop Synthesis Simulation Living Syst., C. G. Langton and K. Shimohara, Eds., Cambridge, MA, 1997, pp. 34-41, MIT Press.

[63] S. A. Huber and H. H. Bulthoff, "Simulation and robot implementation of visual orientation behaviour of flies," in From Animals to Animats 5, R. Pfeifer, B. Blumberg, J. A. Meyer, and S. W. Wilson, Eds Cambridge, MA: MIT Press, 1998, pp. 77-85.

[64] J. Hurford, M. Studdert-Kennedy, and C. Knight, Approaches to the Evolution of Language: Social and Cognitive Bases. Cambridge, MA: Cambridge Univ. Press, 1998.

[65] J. Hurford, "Random boolean nets and features of language," IEEE Trans. Evol. Comput., vol. 5, no. 2, pp. 111-116, Oct. 2001.

[66] P. Husbands and O. Holland, "The ratio club: A hub of British cybernetics," in The Mechanical Mind in History, P. Husband, M. Wheeler, and O. Holland, Eds. Cambridge, MA: MIT Press, 2008.

[67] A. J. Ijspeert, "Central pattern generators for locomotion control in animals and robots: A review," Neural Netw., vol. 21, pp. 642-653, 2008.

[68] F. Kaplan, "Who is afraid of the humanoid? Investigating cultural differences in the acceptance of robots," Int. J. Humanoid Robot., vol. 1, no. 3, pp. 465-480, 2004.

[69] F. Kaplan, La Naissance d'Une Langue Chez Les Robots. Paris, France: Hermes Science, 2001

[70] F. Kaplan and V. V. Hafner, "Information-theoretic framework for unsupervised activity classification," Adv. Robot., vol. 20, no. 10, pp. 1087-1103, 2006

[71] F. Kaplan and P.-Y. Oudeyer, "Stable kernels and fluid body envelopes," SICE J. Control, Measurement, Syst. Integration, vol. 48, no. $1,2009$.

[72] F. Kaplan and P.-Y. Oudeyer, "The progress-drive hypothesis: An interpretation of early imitation," in Models and Mechanisms of Imitation and Social Learning: Behavioural, Social and Communication Dimensions, K. Dautenhahn and C. Nehaniv, Eds. Cambridge, U.K.: Cambridge Univ. Press, 2007, pp. 361-377.

[73] S. Kauffman, At Home in the Universe: The Search for Laws of Self-Organization and Complexity. Oxford, U.K.: Oxford Univ. Press, 1996.

[74] C. C. Kemp and A. Edsinger, "What Can I Control?: The Development of Visual Categories for a Robot's Body and the World that it Influences," in Proc. 5th Int. Conf. Develop. Learn., Special Session Autonom. Mental Develop., 2006.

[75] S. Kirby, "Spontaneous evolution of linguistic structure-an iterated learning model of the emergence of regularity and irregularity," IEEE Trans. Evol. Comput., vol. 5, no. 2, pp. 102-110, Apr. 2001.

[76] A. S. Klyubin, D. Polani, and C. L. Nehaniv, "Empowerment: A universal agent-centric measure of control," in Proc. 2005 IEEE Congress Evol. Comput., 2005, vol. 1, pp. 128-135.

[77] H. Kozima, C. Nakagawa, and Y. Yasuda, "Designing and observing human-robot interactions for the study of social development and its disorders," in Proc. IEEE Int. Symp. Comput. Intell. Robot. Autom., Espoo, Finland, 2005, pp. 41-46.

[78] H. Kozima and C. Nakagawa., "Interactive robots as facilitators of children's social development," Mobile Robots: Toward New Applications, pp. 269-286, 2006.

[79] E. Kubinyi, A. Miklosi, F. Kaplan, M. Gacsi, J. Topal, and V. Csanyi, "Social behaviour of dogs encountering AIBO, an animal-like robot in a neutral and in a feeding situation," Behav. Process., vol. 65, no. 3, pp. 231-239, 2004.

[80] B. Kuipers, P. Beeson, J. Modayil, and J. Provost, "Bootstrap learning of foundational representations," Connect. Sci, vol. 18, no. 2, pp. $145-158,2006$.

[81] D. Lack, "The behaviour of the robin: I and II," Proc. Zool. Soc. Lond. A., vol. 109, pp. 169-178, 1939.

[82] G. Lakoff and M. Johnson, Philosophy in the Flesh: The Embodied Mind and Its Challenge to Western Thought. New York: Basic, 1999.

[83] G. V. Lauder, E. J. Anderson, J. Tangorra, and P. G. A. Madden, "Fish biorobotics: Kinematics and hydrodynamics of self-propulsion," $J$. Exp. Biol., vol. 210, pp. 2767-2780, 2007.

[84] J. Liljencrantz and B. Lindblom, "Numerical simulation of vowel quality systems: The role of perceptual contrast," Language, vol. 48, pp. 839-862, 1972.
[85] B. Lindblom, P. MacNeilage, and M. Studdert-Kennedy, "Self-organizing processes and the explanation of language universals," in Explanations for Language Universals, B. Butterworth, C. Bernard, and O. Dahl, Eds. Berlin, Germany: Mouton, 1984, pp. 181-203.

[86] J.-D. Liu and H. Hu, "Biologically inspired behaviour design for autonomous robotic fish," Int. J. Autom. Comput., vol. 3, pp. 336-347, 2006.

[87] M. Lungarella, G. Metta, R. Pfeifer, and G. Sandini, "Developmental robotics: A survey," Connect. Sci., vol. 15, pp. 151-190, 2003.

[88] M. Lungarella and O. Sporns, "Information self-structuring: Key principle for learning and development," in Proc. 4th Int. Conf. Develop. Learning, 2005, pp. 25-30.

[89] J. Loeb, Comparative Physiology of the Brain and Comparative Psychology. New York: Putnam, 1900.

[90] J. Loeb, Forced movements, tropisms, and animal conduct. London, U.K.: Lippincott, 1918.

[91] J. H. Long, Jr, T. J. Koob, K. Irving, K. Combie, V. Engel, N. Livingston, A. Lammert, and J. Schumacher, "Biomimetic evolutionary analysis: Testing the adaptive value of vertebrate tail stiffness in autonomous swimming robots," J. Exp. Biol., vol. 209, pp. 4732-4746, 2006.

[92] K. H. Low, "Locomotion and depth control of robotic fish with modular undulating fins," Int. J. Autom. Comput., vol. 3, pp. 348-357, 2006.

[93] D. MacKenzie and T. Balch, 1993, Making a Clean Sweep: BehaviorBased Vacuuming Working notes of 1993 AAAI Fall Symposium: Instantiating real-world agents, AAAI. Raleigh, NC.

[94] G. Maimon, A. D. Straw, and M. H. Dickinson, "A simple vision-based algorithm for decision making in flying Drosophila," Curr. Biol., vol. 18 , no. 6, pp. 464-470, 2008.

[95] M. Maris and R. T. Boekhorst, "Exploiting physical constraints: Heap formation through behavioral error in a group of robots," in Proc. IROS '96, Osaka, Japan, Nov. 4-8, 1996.

[96] H. Maturana and F. Varela, Autopoiesis and Cognition: The Realization of the Living. Boston, MA: Reidel, 1980.

[97] J. Mc Carthy, M. Minsky, N. Rochester, and C. Shannon, 1955, A proposal for the Darmouth summer research project on artificial intelligence [Online]. Available: http://www.formal.stanford.edu/jmc/history/dartmouth/dartmouth.html

[98] W. McDougall, Body and mind, a history and a defense of animism. London: Methuen, 1911

[99] T. McGeer, "Passive walking with knees," in Proc. IEEE Robot. Autom Conf., Cincinnati, OH, 1990, pp. 1640-1645.

[100] T. McGeer, "Dynamics and control of bipedal locomotion," J. Theoret. Biol., vol. 16, no. 3, pp. 277-314, 1993.

[101] J. Mehler, A. Christophe, and F. Ramus, "What we know about the initial state for language," in Image, Language, Brain: Papers from the First Mind-Brain Articulation Project Symposium, A. Marantz, Y. Miyashita, and W. O’Neil, Eds. Cambridge, MA: MIT Press, 2000, pp. 51-75.

[102] M. Merleau-Ponty, La Structure Du Comportement. Paris, France: Press Univeristaire, 1942.

[103] M. Merleau-Ponty, Phenomenology of Perception. Evanston, IL: Routledge, 1962.

[104] A. Michelsen, B. Andersen, J. Storm, W. H. Kirchner, and M. Lindauer, "How honeybees perceive communication dances, studied by means of a mechanical model," Behav. Ecol. Sociobiol., vol. 30, pp. 143-150, 1992.

[105] B. F. Miessner, "The wirelessly directed torpedo. Some new experiments in an old field," Sci. Ameri., vol. Jun., p. 53, 1912.

[106] E. M. Mullen, Mullen Scales of Early Learning, AGS Edition ed. Circle Pines, MN: American Guidance Service, 1995.

[107] J. Nadel, A. Revel, P. Andy, and P. Gaussier, "Toward communication, first imitations in infants, low-functioning children with autism and robots," Interaction Stud., vol. 5, no. 1, pp. 45-74, 2004.

[108] A. Newell and H. A. Simon, "Computer science as empirical enquiry: Symbols and search," Commun. ACM, vol. 19, no. 3, pp. 113-126, 1976.

[109] L. Olsson, C. L. Nehaniv, and D. Polani, "Sensory channel grouping and structure from uninterpreted sensor data," in Proc. NASA/DoD Conf. Evolvable Hardware, Seattle, WA, 2004, pp. 153-160.

[110] P.-Y. Oudeyer, "How phonological structures can be culturally selected for learnability," Adapt. Behav., vol. 13, no. 4, pp. 269-280, 2005a.

[111] P.-Y. Oudeyer, "The self-organization of speech sounds," J. Theor Biol., vol. 233, no. 3, pp. 435-449, 2005 b.

[112] P.-Y. Oudeyer, Self-Organization in the Evolution of Speech, Studies in the Evolution of Language. Oxford, U.K.: Oxford Univ. Press, 2006.

[113] P.-Y. Oudeyer and F. Kaplan, "Discovering communication," Connect. Sci., vol. 18, no. 2, pp. 189-206, 2006

[114] P.-Y. Oudeyer and F. Kaplan, "Language evolution as a Darwinian process: Computational studies," Cogn. Process., vol. 8, no. 1, pp. 21-35, 2007. 
[115] P.-Y. Oudeyer and F. Kaplan, "How can we define intrinsic motivation?," in Proc. 8th Int. Conf. Epigenetic Robot.: Modeling Cogn. Develop. Robot. Syst., Brighton, 2008.

[116] P.-Y. Oudeyer, F. Kaplan, and V. Hafner, "Intrinsic motivation systems for autonomous mental development," IEEE Trans. Evol. Comput., vol. 11, no. 2, pp. 265-286, Apr. 2007.

[117] R. Pfeifer and J. C. Bongard, How the Body Shapes the Way We Think-A New View of Intelligence. Cambridge, MA: MIT Press, 2007.

[118] R. Pfeifer, M. Lungarella, and F. Iida, "Self-organization, embodiment, and biologically inspired robotics," Science, vol. 318, pp. 1088-1093, 2007.

[119] R. Pfeifer and C. Scheier, Understanding Intelligence. Cambdrige, MA: MIT Press, 1999.

[120] M. Piattelli-Palmarini, "Evolution, selection and cognition: From "learning" to parameter setting in biology and in the study of language," Cognition, vol. 31, pp. 1-44, 1989.

[121] D. Pierce and B. Kuipers, "Map learning with uninterpreted sensors and effectors," Artif. Intell., vol. 92, pp. 169-229, 1997.

[122] S. Pinker, How the Mind Works. New York: Norton, 2009.

[123] S. Pinker and P. Bloom, "Natural language and natural selection," Behav. Brain Sci., vol. 13.4, pp. 707-726, 1990.

[124] P. Pongrácz and V. Altbäcker, "Ontogeny of the responses of European rabbits (oryctolagus cuniculus) to aerial and ground predators," Can. J. Zool., vol. 78, pp. 655-665, 2000.

[125] D. Premack, "The infant's theory of self-propelled objects," Cognition, vol. 26, pp. 1-16, 1990

[126] H. Putnam, Mind, Language and Reality. Philosophical Papers. Cambridge, MA: Cambridge Univ. Press, 1975, vol. 2.

[127] P. Rochat, The infant's world. Cambridge, MA: Harvard Univ. Press, 2001.

[128] B. Robins and K. Dautenhahn, "Encouraging social interaction skills in children with autism playing with robots: A case study evaluation of triadic interactions involving children with autism, other people (peers and adults) and a robotic toy," Enfance, vol. 1, pp. 72-81, 2007.

[129] A. Robinson-Mosher and B. Scassellati, "Prosody recognition in male infant-directed speech," in Proc. IEEE/RSJ Int. Conf. Intell. Robots Syst., Sendai, Japan, 2004.

[130] A. Samuel, "Some studies in machine learning using the game of checkers," IBM Journal, vol. 3, no. 3, pp. 210-229, 1959.

[131] J. Santos-Victor, G. Sandini, F. Curotto, and S. Garibaldi, "Divergent stereo in autonomous navigation: From bees to robots," Int. J. Comput. Vis., vol. 14, pp. 159-177, 1995.

[132] B. Scassellati, "Using robots to study abnormal social development," in Proc. 5th Int. Workshop Epigenetic Robot., Nara, Japan, 2005.

[133] T. Schatz and P.-Y. Oudeyer, "Learning motor dependent Crutchfield's information distance to anticipate changes in the topology of sensory body maps," in Proc. 8th IEEE Int. Conf. Develop. Learn., Shangai, China, 2009.

[134] J. L. Schwartz, L. J. Boe, N. Valle, and C. Abry, "Major trends in vowel systems inventories," J. Phonetics, vol. 25, pp. 255-286, 1997.

[135] M. V. Srinivasan, M. Lehrer, W. H. Kirchner, and S. W. Zhang, "Range perception through apparent image speed in freely flying honeybee," Visual Neurosci., vol. 6, pp. 519-535, 1991.

[136] M. V. Srinivasan, J. S. Chahl, K. Weber, and S. Venkatesh, "Robot navigation inspired by principles of insect vision," Robot. Autonom. Syst., vol. 26, pp. 203-216, 1999.

[137] C. M. Stanton, P. H. Kahn, Jr., R. L. Severson, J. H. Ruckert, and B. T. Gill, "Robotic animals might aid in the social development of children with autism," in Proc. 3rd ACM/IEEE Int. Conf. Human-Robot Interaction, Amsterdam, The Netherlands, Mar. 12-15, 2008.

[138] L. Steels, "A self-organizing spatial vocabulary," Artif. Life, vol. 2, no. 3, pp. 319-332, 1995.

[139] L. Steels, "The synthetic modeling of language origins," Evol. Commun., vol. 1, 1997.

[140] L. Steels and F. Kaplan, "Bootstrapping grounded word semantics," in Linguistic evolution through language acquisition: Formal and computational models, T. Briscoe, Ed. Cambridge, MA: Cambridge Univ. Press, 2002, pp. 53-74.

[141] L. Steels, "Evolving grounded communication for robots," Trends Cogn. Sci., vol. 7, no. 7, pp. 308-312, 2003.

[142] L. Steels, "Intelligence with representation," Philosoph. Trans. Roy. Soc. A, vol. 361, no. 1811, pp. 2381-2395, 2003.

[143] L. Steels, "The emergence and evolution of linguistic structure: From lexical to grammatical communication systems," Connect. Sci., vol. 17, no. 3-4, pp. 213-230, 2005.

[144] C. Stefanini, G. Orlandi, A. Menciassi, Y. Ravier, G. L. Spina, and S. Grillner et al., "A mechanism for biomimetic actuation in lampreylike robots," in Proc. 1st IEEE/RAS-Embs Int. Conf. Biomed. Robot. Biomech., 2006, pp. 579-584.
[145] K. Stevens, The Quantal nature of speech: Evidence from articulatoryacoustic data. New York: McGraw-Hill, 1972, pp. 51-66.

[146] J. L. Tangorra, S. N. Davidson, I. W. Hunter, P. G. A. Madden, G. V. Lauder, H. Dong, M. Bozkurttas, and R. Mittal, "The development of a biologically inspired propulsor for unmanned underwater vehicles," IEEE J. Ocean. Eng., vol. 32, no. 3, Jul. 2007.

[147] D. Thompson, On Growth and Form, Reprint. Cambridge, MA: Cambridge Univ. Press, 2000, 1917.

[148] M. Tomasello and M. Carpenter, "Shared intentionality," Develop. Sci., vol. 10 , no. 1 , pp. 121-125, 2007.

[149] M. S. Triantafyllou and G. S. Triantafyllou, "An efficient swimming machine," Sci. Amer., vol. 272, pp. 40-48, 1995.

[150] D. P. Tsakiris, M. Sfakiotakis, A. Menciassi, G. La Spina, and P. Dario, "Polychaete-like undulatory robotic locomotion," in Proc. IEEE Int. Conf. Robot. Autom., 2005, pp. 3029-3034.

[151] A. M. Turing, "The chemical basis of morphogenesis," Philosoph Trans. Roy. Soc. London, vol. B 237, pp. 37-72, 1952.

[152] J. R. Usherwood, S. B. Williams, and A. M. Wilson, "Mechanics of dog walking compared with a passive, stiff-limbed, 4-bar linkage model, and their collisional implications," J. Exp. Biol., vol. 210, pp. 533-540, 2007.

[153] F. J. Varela, E. T. Thompson, and E. Rosch, The Embodied Mind: Cognitive Science and Human Experience. Cambridge, MA: MIT Press, 1992.

[154] F. R. Volkmar, C. Lord, A. Bailey, R. T. Schultz, and A. Klin, "Autism and pervasive developmental disorders," J. Child Psychol. Psychiatry, vol. 45 , no. 1, pp. 1-36, 2004.

[155] H. Von Foerster, "Basic concepts of homeostasis," in Homeostatic Mechanisms. Upton, NY: Springer-Verlag, 1958, pp. 216-242.

[156] K. Von Frisch, Bees-Their Vision, Chemical Senses, and Language, Revised Edition ed. Ithaca, NY: Cornell Univ. Press, 1971.

[157] K. Wada, T. Shibata, T. Saito, and K. Tanie, "Analysis of factors that bring mental effects to elderly people in robot assisted activity," in Proc. IEEE/RSJ Int. Conf. Intell. Robot. Syst., 2002, pp. 1152-1157.

[158] W. G. Walter, “A machine that learns," Sci. Amer., vol. 185, pp. 60-63, 1951

[159] G. Walter, The Living Brain. London, U.K.: Penguin, 1953.

[160] G. K. Wallace, "Visual scanning in the desert locust schistocerca gregaria," J. Exper. Biol., vol. 36, pp. 512-525, 1959.

[161] B. Webb, "What does robotics offer animal behaviour?" Animal Behav., vol. 60, pp. 545-558, 2000.

[162] T. Welsh, "Do neonates display innate self-awareness? Why neonatal imitation fails to provide sufficient ground for innate self- and otherawareness," Philosop. Psychol., vol. 19, no. 2, pp. 221-238, 2006.

[163] A. Wenner and P. H. Wells, Anatomy of a Controversy - The Question of a "Language» Among Bees". New York: Columbia Univ. Press, 1990.

[164] I. Werry, K. Dautenhahn, and W. Harwin, "Evaluating the response of children with autism to a robot," in Proc. Rehab. Eng. Assist. Tech. Soc. North Amer. (RESNA), 2001.

[165] C. Wilbur, W. Vorus, Y. Cao, and S. N. Currie, Neurotechnology for Biomimetic Robots, J. Ayers, J. Davis, and A. Rudolph, Eds. Cambridge, MA: MIT Press, 2002, pp. 285-296.

[166] W. Zuidema, "How the poverty of the stimulus solves the poverty of the stimulus," in Advances in Neural Information Processing 15, S. Becker and K. Obermayer, Eds. Cambridge, MA: MIT Press, 2003, pp. 51-68.

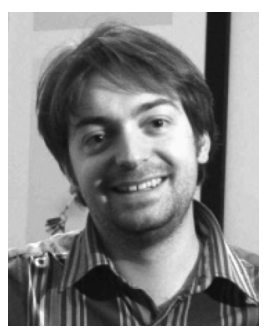

Pierre-Yves Oudeyer received the M.Sc. degree is theoretical computer science from Ecole Normale Supérieure de Lyon, France, and received the Ph.D. degree in artificial intelligence from the University Paris VI, France.

After eight years as a permanent researcher at Sony CSL Paris, he is now a Research Scientist at INRIA, France, where he heads the FLOWERS team. He has published a book, more than 60 papers in international journals and conferences, and received several prizes for his work in developmental robotics and on the origins of language. He is interested in the mechanisms that allow humans and robots to develop perceptual, motivational, behavioral, and social capabilities to become capable of sharing cultural representations.

Dr. Oudeyer is Editor of the IEEE CIS NEWSLETTER ON AUTONOMOUS Mental Development, and Associate Editor of IEEE Transactions on Autonomous Mental Development, Frontiers in Neurorobotics, and of the International Journal of Social Robotics. 